\title{
Potencial inflamatório da dieta e risco de câncer de mama
}

\author{
Inflammatory potential of the diet and risk of breast cancer
}

Potencial inflamatorio de la dieta y riesgo de cáncer de mama

Thaís Rodrigues Nogueira ${ }^{1 *}$, Daniele Rodrigues Carvalho Caldas², Camila Guedes Borges de Araújo $^{3}$, Maria da Cruz Moura e Silva ${ }^{4}$, Nadir do Nascimento Nogueira ${ }^{5}$, Gilmara Péres Rodrigues ${ }^{6}$.

\section{RESUMO}

Objetivo: Revisar o conhecimento atual sobre o potencial inflamatório da dieta e o risco de neoplasia mamária. Métodos: Trata-se de revisão integrativa da literatura, realizada por meio de pesquisa de artigos originais, publicados nos últimos dez anos, em inglês, português e espanhol, indexados nas bases de dados Pubmed e Scopus (Elsevier $\left.{ }^{\circledR}\right)$. Resultados: Foram recuperados 93 artigos, dos quais $11,8 \%(n=11)$ foram incluídos nesta revisão, para leitura do texto integral. Após análise do texto completo, verificou-se que $81,8 \%$ $(n=9)$ demonstraram relação positiva entre a adoção de padrões alimentares pró-inflamatórios e o risco aumentado de câncer de mama na população feminina. De forma contrária, dois artigos $(18,2 \%)$ demonstraram que o padrão dietético não é fator determinante da carcinogênese mamária, embora possa potencializar o desenvolvimento da doença em mulheres com predisposição genética. Conclusão: Concluise que dietas de elevado potencial inflamatório estão associadas ao aumento do risco de câncer de mama.

Palavras-chave: Índice, Inflamação, Dietas, Câncer de mama.

\begin{abstract}
Objective: To review the current knowledge about the inflammatory potential of the diet and the risk of breast neoplasia. Methods: This is an integrative review of the literature, carried out by searching for original articles published in the last ten years in English, Portuguese and Spanish, indexed in Pubmed and Scopus (Elsevier ${ }^{\circledR}$ ) databases. Results: 93 articles were retrieved, of which $11.8 \%(n=11)$ were included in this review, for reading the full text. After analysis of the full text, it was verified that $81.8 \%(n=9)$ demonstrated a positive relationship between the adoption of pro-inflammatory dietary patterns and the increased risk of breast cancer in the female population. Conversely, two articles (18.2\%) demonstrated that the dietary pattern is not a determinant of mammary carcinogenesis, although it may potentiate the development of the disease in women with genetic predisposition. Conclusion: It is concluded that diets with high inflammatory potential are associated with an increased risk of breast cancer.
\end{abstract}

Keywords: Index, Inflammation, Diets, Breast Cancer.

\footnotetext{
${ }^{1}$ Pós-Graduanda em Obesidade e Emagrecimento, Universidade Cândido Mendes, Rio de Janeiro, Rio de Janeiro, Brasil. E-mail: thaisnogueiranutri@gmail.com

${ }^{2}$ Professora Assistente da Faculdade de Ciências e Tecnologia do Maranhão, FACEMA, Caxias, Maranhão, Brasil.

${ }^{3}$ Professora Titular da Faculdade Pitágoras, Bacabal, Maranhão, Brasil.

${ }^{4}$ Nutricionista do Hospital Universitário, Universidade Federal do Piauí, Teresina, Piauí, Brasil.

${ }^{5}$ Professora Titular do Departamento de Nutrição, Universidade Federal do Piauí, Teresina, Piauí, Brasil.

${ }^{6}$ Professora Adjunto do Departamento de Nutrição, Universidade Federal do Piauí, Teresina, Piauí, Brasil.
}

SUBMETIDO EM: 11/2018

ACEITO EM: 12/2018

PUBLICADO EM: 4/2019

REAS/EJCH | Vol.Sup.22 | e571 | DOI: https://doi.org/10.25248/reas.e571.2019 Página 1 de 8 


\section{RESUMEN}

Objetivo: Revisar el conocimiento actual sobre el potencial inflamatorio de la dieta y el riesgo de neoplasia. Métodos: Se trata de una revisión integrativa de la literatura, realizada por medio de la investigación de artículos originales, publicados en la última década en inglés, portugués y español, indexados en las bases de los dados de Pubmed e Scopus (Elsevier®). Resultados: Se han recuperado 93 artículos, de los cuales $11,8 \%(n=11)$ se incluyeron en esta revisión, para la lectura del texto completo. Después del análisis del texto completo, se verificó que $81,8 \%(n=9)$ demostraron una relación positiva entre la adopción de patrones alimentarios pro-inflamatorios y el riesgo aumentado de cáncer de mama en la población femenina. Por otro lado, dos artículos $(18,2 \%)$ demostraron que el patrón dietético no es factor determinante de la carcinogénesis mamaria, aunque puede potenciar el desarrollo de la enfermedad en mujeres con predisposición genética. Conclusión: Se concluye que las dietas de alto potencial inflamatorio están asociadas al aumento del riesgo de cáncer de mama.

Palabras clave: Índice, Inflamación, Dietas, Cáncer de mama.

\section{INTRODUÇÃO}

O câncer é um problema de saúde pública, apontado como a $3^{\text {a }}$ causa de morte em todo o mundo, o que equivale a aproximadamente 6 milhões de óbitos a cada ano (OMS, 2004). No Brasil, com exceção dos tumores de pele não melanoma, o câncer de mama é o de maior incidência na população feminina, estimandose 59.700 casos novos desta neoplasia, para cada ano do biênio 2018-2019 (INCA, 2017).

A etiologia do câncer de mama é multifatorial, e permanece não elucidada. A interação entre fatores de risco ambientais e o perfil genético individual pode inibir ou favorecer o início e desenvolvimento do processo carcinogênico. Nas últimas décadas, destaque tem sido atribuído à influência dos padrões alimentares e de seus constituintes dietéticos na modulação do risco de vários cânceres, por seu impacto e possibilidade de intervenção (GE et al., 2015; SHIVAPPA et al., 2015; WIRTH et al., 2015).

O consumo excessivo de alimentos industrializados, ricos em calorias, gorduras saturadas, ácidos graxos trans, açúcares simples e sódio, somado ao consumo reduzido de verduras, legumes e frutas, foi demonstrado como padrão alimentar favorável ao desenvolvimento do câncer de mama (TORRES et al., 2015). Em geral, esse padrão alimentar, aliado ao sedentarismo, são as principais características do estilo de vida ocidental, e estão relacionados à obesidade, neoplasias, diabetes melito, doenças cardiovasculares e neurodegenerativas. Em todas essas afecções, evidencia-se como base fisiopatológica comum, a inflamação subclínica, também denominada inflamação sistêmica de baixo grau, caracterizada pela síntese e aumento nos níveis circulantes de adipocitocinas, em decorrência do tecido adiposo em excesso. Nesse processo, os principais envolvidos são as interleucinas 6 (IL-6), 8 (IL-8) e $1 \beta$ (IL-1 $\beta$ ), o fator de necrose tumoral- $\alpha$ (TNF- $\alpha$ ) e os receptores de membrana CD40/CD40L, responsáveis por sinalizar e/ou modular o estado de inflamação de baixo grau, além de promover o desenvolvimento de desordens clínicas. É importante destacar que a inflamação subclínica tem efeitos a longo prazo, diferenciando-se do processo clássico de inflamação, que ocorre em resposta à lesão vascular ou tecidual, de forma aguda ou crônica, com atuação discreta (resposta aguda) ou intensificada (crônica) do sistema imunológico (PRADO et al., 2009; SHIVAPPA et al., 2014a; WU e WU, 2006)

Nesse contexto, é oportuno considerar que os alimentos regularmente consumidos, com seus nutrientes e compostos bioativos, estabelecem um elo com marcadores de inflamação, permitindo atribuir à dieta habitual um caráter pró ou anti-inflamatório (JAKOBSEN et al., 2010). Nos últimos anos, tem sido possível confirmar a relação entre dieta e inflamação por meio do cálculo do Índice Inflamatório da Dieta ou Dietary Inflammatory Index (DII ${ }^{\circledR}$ ) (CAVICCHIA et al., 2009; SHIVAPPA et al., 2013). 
Considerando a relação entre alimentação e marcadores inflamatórios na fisiopatologia de diversas doenças crônicas, este estudo tem como objetivo revisar o conhecimento atual sobre o potencial inflamatório da dieta e o risco de câncer de mama.

\section{METODOLOGIA}

Trata-se de revisão integrativa da literatura, realizada por meio de pesquisas bibliográficas de estudos originais, publicados nos últimos dez anos e indexados às bases de dados PubMed e Scopus (Elsevier $\left.{ }^{\circledR}\right)$, cujo objetivo relacionava o potencial inflamatório da dieta ao risco de câncer de mama. As palavras-chave foram escolhidas entre os Descritores em Ciências da Saúde (DeCS: http://decs.bvs.br): index (índice), inflammation (inflamação), diets (dietas) e "breast cancer" (câncer de mama).

Como critérios de elegibilidade dos estudos, foram estabelecidos: idioma de publicação: português, inglês ou espanhol, adequação ao problema de pesquisa, ano de publicação no período de 2008 a 2018, e desenho de estudo: caso controle, experimental ou ensaio clínico. Foram excluídas publicações não originais, revisões sistemáticas e metanálise, bem como artigos originais disponíveis apenas no formato de resumo ou repetidos.

Foram encontrados 93 artigos, dos quais $11,8 \%(n=11)$ foram incluídos nesta revisão para leitura do texto completo e $16,1 \%(n=15)$ foram excluídos, por serem repetidos. Somente um dos artigos que avaliou 0 potencial inflamatório da dieta em mulheres com câncer de mama foi excluído, pois seu objetivo relacionou o DII ${ }^{\circledR}$ à mortalidade por câncer de mama e não ao risco de desenvolver a doença (ZUCCHETTO et al., 2017).

\section{DISCUSSÃO}

\section{Índice Inflamatório da Dieta $\left(D^{\circledR}\right)$}

Diversos são os índices alimentares utilizados em estudos científicos para auxiliar pesquisadores e nutricionistas a caracterizar a qualidade da dieta habitual de um indivíduo ou população, associando-a ao desenvolvimento de Doenças Crônicas Não Transmissíveis (DCNT). Porém, a proposta inovadora de pesquisadores da Universidade da Carolina do Sul, integrantes do Programa de Prevenção e Controle do Câncer, nos Estados Unidos, foi relacionar dieta e inflamação, desenvolvendo, em 2009, o Índice Inflamatório da Dieta ou Dietary Inflammatory Index (DII ${ }^{\circledR}$ (CAVICCHIA et al., 2009).

O DII ${ }^{\circledR}$ é a primeira técnica de avaliação fundamentada e validada, tendo a inflamação como principal objeto de estudo (GUENTHER et al., 2007; KWAN et al., 2009). Esse índice reconhece a dieta como um conjunto complexo e representativo de exposições que interagem, produzindo respostas inflamatórias capazes de promover repercussões à saúde (SHIVAPPA et al., 2013).

Inicialmente elaborado e validado por Cavicchia et al. (2009), como uma proposta de instrumento para avaliação do potencial inflamatório da dieta, a versão atual do DII ${ }^{\circledR}$ foi modificada por Shivappa et al. (2014a), considerando dados dietéticos de inquéritos alimentares de 11 países e o efeito inflamatório global de parâmetros alimentares específicos.

Nesse contexto, é oportuno mencionar que, para sua proposta inicial, Cavicchia et al. (2009) realizaram estudo de base populacional, no qual observaram a associação do $D I^{\circledR}$ às concentrações séricas de Proteína C-Reativa (PCR), propondo escores variáveis de maximamente anti-inflamatório (- 8 pontos) a maximamente pró-inflamatório (+ 8 pontos). Posteriormente, para uma proposta atualizada, Shivappa et al. (2014b) utilizaram a PCR ultrassensível como marcador inflamatório para modificação e validação do $\mathrm{DI}^{\circledR}$ a partir de dados do estudo longitudinal Seasonal Variation of Blood Cholesterol Study (SEASONS), realizado em Worcester, nos Estados Unidos. Esses pesquisadores construíram a versão atual do DII ${ }^{\circledR}$, por meio de uma vasta revisão da literatura, considerando o período de 1950 a 2010, para o qual avaliaram 1943 artigos, os quais receberam, cada um, pontuação referente ao efeito do parâmetro alimentar sobre a inflamação: +1 , para indicar efeito pró-inflamatório; -1 , para indicar efeito anti-inflamatório; e 0 , para indicar ausência de efeito sobre a inflamação. 
$\mathrm{Na}$ atualização realizada em 2014, o objetivo foi avaliar o efeito de 45 parâmetros alimentares sobre as concentrações dos marcadores inflamatórios: interleucina 1 beta (IL-1 $\beta$ ), interleucina 4 (IL-4), interleucina 6 (IL-6), interleucina 10 (IL-10), fator de necrose tumoral alfa (TNF- $\alpha$ ) e PCR. Os 45 parâmetros alimentares incluíram os seguintes alimentos, nutrientes e compostos bioativos: alecrim, alho, açafrão, ácido fólico, ácidos graxos monoinsaturados e poliinsaturados, antocianidinas, ácidos graxos saturados, ácidos graxos trans, álcool, $\beta$-caroteno, cafeína, carboidratos, cebola, chás verde e preto, colesterol, energia, eugenol, ferro, fibras, flavan-3-ol, flavonóis, flavonas, flavononas, gengibre, gorduras totais, isoflavonas, magnésio, ômega-3, ômega-6, orégano, pimenta, proteínas, riboflavina, saffron, selênio, zinco, niacina, tiamina, vitaminas $A, B 6$, B12, C, D e E (SHIVAPPA et al., 2014a).

Nesse sentido, a aplicação do $\mathrm{D} \|{ }^{\circledR}$ para avaliar o potencial inflamatório da dieta habitual de indivíduos ou populações requer a obtenção de dados referentes aos alimentos consumidos e suas quantidades. Para essa finalidade, os inquéritos alimentares, tais como o questionário de frequência alimentar semi-quantitativo (QFASQ), o recordatório de 24, 48 ou 72 horas (R24, R48, R72) e o registro alimentar de 3 dias (RA) são preferencialmente aplicados (RAMALLAL et al., 2015).

\section{Padrão Inflamatório da Dieta e Câncer de Mama}

O padrão alimentar pode apresentar um caráter inflamatório ou anti-inflamatório, a depender dos seus componentes dietéticos. Estudos apontam que o padrão alimentar anti-inflamatório, caracterizado pela elevada ingestão de peixes, frutas, cereais, vegetais crus, oleaginosas, e, portanto, das vitaminas E e D, betacarotenos, flavanóides, fibras, ácidos graxos monoinsaturados e poli-insaturados, está associado negativamente ao risco de câncer de mama. Por outro lado, o padrão alimentar pró-inflamatório, típico da dieta ocidental, tem associação positiva com a carcinogênese mamária, caracterizando-se principalmente pela ingestão de pães, massas, carnes vermelha e processada, embutidos, produtos alimentícios refinados e açúcares simples (SOUZA, 2014; TORRES et al., 2015).

O uso do DII ${ }^{\circledR}$ como indicador do potencial inflamatório da dieta e sua relação com o aumento do risco carcinogênico tem recebido destaque em estudos publicados em anos recentes (LIU et al., 2017; SHIVAPPA et al., 2017a; SHIVAPPA et al., 2017b; TABUNG et al., 2017). A inflamação, além de representar a resposta fisiológica do corpo a uma lesão tecidual ou a estímulos inflamatórios, representa um fator de risco conhecido para vários tipos de neoplasias, incluindo o câncer de mama (SHIVAPPA et al., 2015).

Na presente revisão de literatura, do total de 11 estudos analisados, $81,8 \%(n=9)$ demonstraram relação positiva entre a adoção de padrões alimentares pró-inflamatórios e o risco aumentado de câncer de mama na população feminina. Em contrapartida, dois artigos $(18,1 \%)$ demonstraram que o padrão dietético não é fator determinante para neoplasia mamária (Tabela 1).

O conhecimento sobre a relação entre carcinogênese mamária e o potencial inflamatório da dieta ainda não está completamente esclarecido. Na carcinogênese, estudos demonstraram aumento nos níveis séricos de marcadores inflamatórios, como as citocinas e quimiocinas, que sinalizam um status de inflamação subclínica e formam um microambiente favorável à iniciação tumoral, crescimento e invasão metastática (IMAYAMA et al., 2012; SIMONE et al., 2018).

Esse status de inflamação subclínica tem atuação intensificada na fisiopatologia do câncer de mama, quando associado a outros fatores de risco, como as alterações hormonais que ocorrem no período menopáusico e o excesso de peso corporal. Nessas condições, o potencial pró-inflamatório da dieta, indicado por escores elevados de $\mathrm{DI}^{\circledR}$, parece aumentar o risco de desenvolvimento de câncer de mama, principalmente em mulheres obesas na pós-menopausa (SHIVAPPA et al., 2016).

Em sua maioria, as dietas pró-inflamatórias seguem o padrão alimentar ocidental, sendo hipercalóricas, hiperlipídicas e favoráveis ao ganho de peso e obesidade. Dietas habituais hipercalóricas estimulam a síntese do IGF-1 (fator de crescimento semelhante à insulina 1) e a ativação do seu receptor (IGF-1R). Esse receptor possui propriedade tirosina quinase que catalisa a fosforilação de proteínas envolvidas em vias inflamatórias e de proliferação celular, como a MAPK (proteína quinase mitógeno-ativada), crescimento celular, translação e inibição da apoptose (CASTRO e GUERRA-JÚNIOR, 2005; GROOT et al., 2016). 
Tabela 1. Principais conclusões sobre a relação entre o potencial inflamatório da dieta e o risco de carcinogênese mamária.

\begin{tabular}{|c|c|c|c|c|c|}
\hline $\begin{array}{l}\text { Autor } \\
\text { (ano) }\end{array}$ & $\begin{array}{l}\text { Desenho de } \\
\text { Estudo }\end{array}$ & $\begin{array}{c}\text { Tamanho } \\
\text { da Amostra }\end{array}$ & $\begin{array}{l}\text { Idade } \\
\text { (anos) }\end{array}$ & Variáveis Estudadas & Conclusões \\
\hline $\begin{array}{c}\mathrm{Ge} \\
\text { et al. (2015) }\end{array}$ & Caso-controle & 8.399 & $50-74$ & $\begin{array}{l}\text { Fatores de risco para câncer de mama } \\
\text { (clínicos, hormonais, reprodutivos, } \\
\text { familiares e de estilo de vida); consumo } \\
\text { alimentar; índice de massa corporal (IMC). }\end{array}$ & $\begin{array}{l}\text { Os achados podem refletir uma ausência real } \\
\text { de associação entre o potencial inflamatório da } \\
\text { dieta e o risco de câncer de mama na pós- } \\
\text { menopausa ou a subestimação da associação } \\
\text { devido ao viés de memória. }\end{array}$ \\
\hline $\begin{array}{l}\text { Shivappa } \\
\text { et al. (2015) }\end{array}$ & Coorte & 45.257 & $\begin{array}{c}\text { (Não } \\
\text { especificada) }\end{array}$ & $\begin{array}{l}\text { Fatores de risco para câncer de mama } \\
\text { (clínicos, hormonais, reprodutivos, } \\
\text { familiares e de estilo de vida); consumo } \\
\text { alimentar; uso de multivitamínicos e altura. }\end{array}$ & $\begin{array}{l}\text { A dieta pró-inflamatória parece aumentar o } \\
\text { risco de desenvolver câncer de mama, } \\
\text { especialmente em mulheres na pós- } \\
\text { menopausa. }\end{array}$ \\
\hline $\begin{array}{l}\text { Shivappa } \\
\text { et al. (2017a) }\end{array}$ & Caso-controle & 5.157 & $20-74$ & $\begin{array}{l}\text { IMC, paridade, estado menopausal, } \\
\text { consumo alimentar; história familiar para } \\
\text { câncer de mama. }\end{array}$ & $\begin{array}{l}\text { A dieta pró-inflamatória está associada ao } \\
\text { aumento do risco de câncer de mama. }\end{array}$ \\
\hline $\begin{array}{l}\text { Shivappa } \\
\text { et al. (2017b) }\end{array}$ & $\begin{array}{c}\text { Coorte } \\
\text { Prospectivo }\end{array}$ & 33.817 & $55-69$ & $\begin{array}{l}\text { Fatores de risco para câncer de mama } \\
\text { (clínicos, hormonais, reprodutivos e de estilo } \\
\text { de vida); consumo alimentar; IMC e } \\
\text { escolaridade. }\end{array}$ & $\begin{array}{l}\text { A dieta pró-inflamatória, como indicado pelos } \\
\text { maiores escores de DII }{ }^{\Theta} \text {, parece aumentar o } \\
\text { risco de desenvolver câncer de mama, } \\
\text { especialmente em mulheres obesas na pós- } \\
\text { menopausa. }\end{array}$ \\
\hline $\begin{array}{l}\text { Haung } \\
\text { et al. (2017) }\end{array}$ & Caso-controle & 1.691 & $25-70$ & $\begin{array}{l}\text { IMC, raça ou etnia, atividade física, consumo } \\
\text { alimentar, nível educacional, tabagismo, } \\
\text { história reprodutiva, estado menopausal, } \\
\text { história familiar de câncer de mama. }\end{array}$ & $\begin{array}{l}\text { Escores elevados de } \mathrm{DII}^{\circledR} \text {, correspondendo a } \\
\text { dietas mais pró-inflamatórias, foram } \\
\text { positivamente associados ao risco de câncer } \\
\text { de mama. }\end{array}$ \\
\hline $\begin{array}{l}\text { Tabung } \\
\text { et al. (2016) }\end{array}$ & $\begin{array}{c}\text { Coorte } \\
\text { Prospectivo }\end{array}$ & 122.788 & $50-79$ & $\begin{array}{l}\text { IMC, raça ou etnia, atividade física, nível } \\
\text { educacional, tabagismo, consumo } \\
\text { alimentar, história familiar de câncer de } \\
\text { mama, uso de anti-inflamatório não } \\
\text { esteroide (AINE). }\end{array}$ & $\begin{array}{l}\text { O potencial inflamatório da dieta antes do } \\
\text { diagnóstico está relacionado à morte por } \\
\text { câncer de mama. }\end{array}$ \\
\hline
\end{tabular}

REAS/EJCH | Vol.Sup.22 | e571 | DOI: https://doi.org/10.25248/reas.e571.2019 Página 5 de 8 


\begin{tabular}{|c|c|c|c|c|c|}
\hline $\begin{array}{c}\text { Tabung } \\
\text { et al. (2017) }\end{array}$ & $\begin{array}{c}\text { Coorte } \\
\text { Prospectivo }\end{array}$ & 70.988 & $50-79$ & $\begin{array}{l}\text { IMC, raça ou etnia, atividade física, consumo } \\
\text { alimentar, nível educacional, tabagismo, } \\
\text { história familiar de câncer de mama. }\end{array}$ & $\begin{array}{l}\text { O potencial inflamatório da dieta influencia, de } \\
\text { modo diferenciado, o desenvolvimento de } \\
\text { fenótipos específicos de câncer de mama. }\end{array}$ \\
\hline $\begin{array}{l}\text { Gardeazabal } \\
\text { et al. (2018) }\end{array}$ & $\begin{array}{c}\text { Coorte } \\
\text { Prospectivo }\end{array}$ & 10.713 & $\begin{array}{l}\text { "mulheres de } \\
\text { meia-idade" }\end{array}$ & $\begin{array}{l}\text { IMC, raça/etnia, atividade física, consumo } \\
\text { alimentar, nível educacional, prática de } \\
\text { amamentação, reposição hormonal, } \\
\text { tabagismo, história familiar de câncer de } \\
\text { mama. }\end{array}$ & $\begin{array}{l}\text { Não houve associação significativa entre } \\
\text { potencial inflamatório da dieta e ocorrência de } \\
\text { câncer de mama. }\end{array}$ \\
\hline $\begin{array}{c}\text { Jalali } \\
\text { et al. (2018) }\end{array}$ & Caso-controle & 408 & 30 & $\begin{array}{l}\text { IMC, raça/etnia, atividade física, consumo } \\
\text { alimentar, nível educacional, tabagismo, } \\
\text { história familiar de câncer de mama. }\end{array}$ & $\begin{array}{l}\text { Dietas pró-inflamatórias podem aumentar o } \\
\text { risco de câncer de mama entre mulheres na } \\
\text { pré-menopausa. }\end{array}$ \\
\hline $\begin{array}{c}\text { Jang } \\
\text { et al. (2018) }\end{array}$ & $\begin{array}{c}\text { Coorte } \\
\text { Prospectivo }\end{array}$ & 511 & $\begin{array}{c}\text { (idade pré- } \\
\text { menopausa }<50 \\
\text { anos e pós- } \\
\text { menopausa: } \geq 50 \\
\text { anos) }\end{array}$ & $\begin{array}{l}\text { IMC, raça/etnia, atividade física, consumo } \\
\text { alimentar, nível educacional, tabagismo, } \\
\text { história familiar de câncer de mama. }\end{array}$ & $\begin{array}{l}\text { Dietas pro-inflamatórias foram positivamente } \\
\text { associadas ao risco de recidiva e mortalidade } \\
\text { por câncer de mama, de acordo com fatores } \\
\text { como idade }(<50 \text { anos }) \text { estado pré- } \\
\text { menopáusico, IMC e características do tumor. }\end{array}$ \\
\hline $\begin{array}{c}\text { Vahid } \\
\text { et al. (2018) }\end{array}$ & Caso-controle & 293 & $20-80$ & $\begin{array}{l}\text { IMC, atividade física, nível educacional, } \\
\text { tabagismo, consumo alimentar, história } \\
\text { familiar de câncer de mama, estado } \\
\text { menopausal, situação conjugal, emprego, } \\
\text { número de filhos. }\end{array}$ & $\begin{array}{l}\text { Dietas pró-inflamatórias foram associadas ao } \\
\text { aumento do risco de câncer de mama, quando } \\
\text { comparadas às dietas anti-inflamatórias. }\end{array}$ \\
\hline
\end{tabular}

Fonte: Dados da Pesquisa, 2018.

REAS/EJCH | Vol.Sup.22 | e571 | DOI: https://doi.org/10.25248/reas.e571.2019 Página 6 de 8 
Dietas hiperlipídicas, ricas em gorduras saturadas, aumentam o risco de câncer de mama pelo estímulo à síntese de citocinas inflamatórias, especialmente a IL-6, envolvida na via da proteína STAT3 (transdutores de sinal e ativadores de transcrição 3), responsável pela inibição da apoptose e aumento da proliferação celular no tecido mamário (SHIVAPPA et al., 2017a).

Nesta revisão, os estudos desenvolvidos por Ge et al. (2015) e Gardeazabal et al. (2018) não demonstraram associação entre o potencial inflamatório da dieta e o risco de câncer de mama em mulheres na pós-menopausa. Apesar disso, é importante mencionar que Ge et al. (2015) não descartaram a existência dessa associação, pois os dados alimentares obtidos para avaliação do DI $^{\circledR}$ foram referentes ao período de um ano, que pode ser um curto espaço de tempo para representar adequadamente uma dieta pró-inflamatória habitual e de efeito crônico. Em acréscimo, Gardeazabal et al. (2018) atribuíram os seus resultados ao pequeno número de casos observados ( $\mathrm{n}=100$ diagnósticos) na coorte de 10.713 universitárias espanholas, acompanhadas pelo período de 10,3 anos.

Em adição, referente à interação entre dieta e genótipo individual, Tabung et al. (2017) sugerem que o potencial inflamatório da dieta tem influência diferenciada para o risco de câncer de mama, de acordo com os diversos subtipos moleculares identificados para a doença. No estudo mencionado, dietas altamente próinflamatórias apresentaram contribuição de risco superior a $100 \%$ em mulheres com tumores do subtipo molecular HER2 superexpresso, caracterizado por negatividade dos receptores de estrogênio (ER-) e progesterona (PR-), com positividade do receptor tipo 2 do fator de crescimento epidérmico humano (HER2++). Por outro lado, o aumento do risco de câncer de mama não foi observado entre as mulheres com tumores do subtipo molecular triplo negativo (ER-, PR- e HER2-) que consumiam dietas pró-inflamatórias (TABUNG et al., 2017).

Considerando os aspectos abordados, destaca-se a importância do potencial inflamatório da dieta, seja anti-inflamatório estável, neutro ou pró-inflamatório estável, na carcinogênese, pois a contribuição causal deste fator de risco para o desenvolvimento do câncer de mama tem caráter modificável.

\section{CONCLUSÃO}

Os estudos avaliados nesta revisão demonstram que o potencial inflamatório da dieta representa um importante fator de risco para a carcinogênese mamária, principalmente em mulheres na pós-menopausa e com obesidade, exercendo influência diferenciada de acordo com o subtipo molecular do tumor.

\section{CONFLITO DE INTERESSES}

Os autores declaram que não existem conflitos de interesses.

\section{REFERÊNCIAS}

1. CAVICCHIA PP, STECK SE, HURLEY TG et al. A new dietary inflammatory index predicts interval changes in high-sensitivity creactive protein. Journal Nutrition, 2009; 139(12): 2365-2372.

2. CASTRO AMS, GUERRA-JÚNIOR G. GH/IGF e Neoplasia: O que há de novo nesta associação? Arquivos Brasileiros de Endocrinologia \& Metabologia, 2005; 49(5): 833-842.

3. COUSSENS L, WERB Z. Inflammation and cancer. Nature, 2002; 420(6917): 860-867.

4. DARVALL KAL, SAM RC, SILVERMAN SH et al. Obesity and thrombosis. European Journal of Vascular and Endovascular Surgery, 2007; 33(2): 223-33.

5. GARDEAZABAL I, RUIZ-CANELA, M., SÁNCHEZ-BAYONA R et al. Dietary inflammatory index and incidence of breast cancer in the SUN Q6 project. Clinical Nutrition, 2018; 18: 1-10.

6. GE I, RUDOLPH A, SHIVAPPA N et al. Dietary inflammation potential and postmenopausal breast cancer risk in a German casecontrol study. The Breast, 2015; 24(4): 491-496.

7. GROOT S, CHAREHBILI A, VAN LAARHOVEN HWM et al. Insulin-like growth factor 1 receptor expression and IGF1R 3129G> T polymorphism are associated with response to neoadjuvant chemotherapy in breast cancer patients: results from the NEOZOTAC trial (BOOG 2010-01). Breast Cancer Research, 2016; 18(3): 1-11.

8. GUENTHER PM, REEDY J, KREBS-SMITH SM et al. Development and evaluation of the Healthy Eating Index-2005: Technical report. Center for Nutrition Policy and Promotion, US Department of Agriculture, 2007; 1-41. 
9. HUANG WQ, MO XF, Y YB et al. A higher Dietary Inflammatory Index score is associated with a higher risk of breast cancer among Chinese women: A case-control study. British Journal of Nutrition, 2017; 117(10): 1358-1367.

10. IMAYAMA I, ULRICH CM, ALFANO CM et al. Effects of a caloric restriction weight loss diet and exercise on inflammatory biomarkers in overweight/obese postmenopausal women: a randomized controlled trial. Effects of a caloric restriction weight loss diet and exercise on inflammatory biomarkers in overweight/obese postmenopausal women: a randomized controlled trial. Cancer Research, 2012; 72(9): 2314-2326.

11. INSTITUTO NACIONAL DE CÂNCER. José Alencar Gomes da Silva. Ministério da saúde. Coordenação de Prevenção e Vigilância Estimativa 2016: Incidência de Câncer no Brasil / Instituto Nacional de Câncer José Alencar Gomes da Silva, Coordenação de Prevenção e Vigilância. Rio de Janeiro: INCA, 2017.

12. JALALI S, SHIVAPPA N, HÉBERT JR et al. Dietary Inflammatory Index and Odds of Breast Cancer in a Case-Control Study from Iran. Nutrition and Cancer, 2018; 20: 1-9.

13. JANG H, CHUNG MS, KANG SS et al. Association between the dietary inflammatory index and risk for cancer recurrence and mortality among patients with breast cancer. Nutrients, 2018; 10(8): 1095.

14. JAKOBSEN MU, DETHLEFSEN C, JOENSEN AM et al. Intake of carbohydrates compared with intake of saturated fatty acids and risk of myocardial infarction: importance of the glycemic index. The American Journal of Clinical Nutrition, 2010; 91(6): 1764-1768.

15. KWAN ML, WELTZIEN E, KUSHI LH et al. Dietary patterns and breast cancer recurrence and survival among women with early-stage breast cancer. Journal Clinical Oncology, 2009; 27(6): 919-26.

16. LAHOZA C, MOSTAZAA JM. Atherosclerosis as a systemic disease. Revista Española de Cardiologia, 2007; 60(2): 184-95.

17. LIU L, NISHIHARA R, QIAN ZR et al. Association between inflamamatory diet pattern and risk of colorectal carcinoma subtypes classified by immune responses to tumor. Gastroenterology, 2017; 153(6): 1517-1530.

18. PRADO WL, LOFRANO MC, OYAMA LM et al. Obesidade e Adipocinas Inflamatórias: Implicações Práticas para a Prescrição de Exercício. Rev Bras Med Esporte. 2009; 15(5):378 -383.

19. RAMALLAL R, TOLEDO E, MARTÍNEZ-GONZÁLEZ MA et al. Dietary Inflammatory Index and Incidence of Cardiovascular Disease in the SUN Cohort. PLOSONE, 2015; 10(9): 1-15.

20. SHIVAPPA N, BOSETTI C, ZUCCHETTO A et al. Association between dietary inflammatory index and prostate cancer among Italian men. British Journal of Nutrition, 2015; 113(2): 278-283.

21. SHIVAPPA N, STECK SE, HURLEY TG et al. Designing and developing a literature-derived, population-based dietary inflammatory index. Public Health Nutrition, 2014a; 17(8): 1689-1696.

22. SHIVAPPA N, STECK SE, HURLEY TG et al. A population-based dietary inflammatory index predicts levels of C-reactive protein in the Seasonal Variation of Blood Cholesterol Study (SEASONS). Public Health Nutrition, 2014b; 17(8); 1825-1833.

23. SHIVAPPA N, SANDIN S, LOF M et al. Prospective study of dietary inflammatory index and risk of breast cancer in Swedish women, British Journal of Cancer, 2015; 113(7): 1099-1103.

24. SHIVAPPA N, HÉBERT JR, ROSATO V et al. Association between the dietary inflammatory index and breast cancer in a large Italian case-control study. Mol Nutr Food Res. March, v.61, n.3, 2017a.

25. SHIVAPPA N, BLAIR CK, PRIZMENT AE et al. Prospective study of the dietary inflammatory index and risk of breast cancer in postmenopausal women. Molecular Nutrition \& Food Research, 2017b; 61(5): 1-18.

26. SIMONE BA, PALAGANI A, STRICKLAND $K$ et al. Caloric restriction counteracts chemotherapy-induced inflammation and increases response to therapy in a triple negative breast cancer model. Cell Cycle, 2018; 17(13): 1536-1544.

27. SOUZA MRGD. Avaliação do uso de ômega 3 em pacientes oncológicos: uma revisão de literatura. Monografia (Bacharelado em Nutrição). Universidade Federal da Paraíba, João Pessoa - Paraíba, 2014; 43p.

28. TABUNG FK, STECK SE, LIESE AD et al. Association between dietary inflammatory potential and breast cancer incidence and death: results from the Women's Health Initiative. British Journal of Cancer, 2016; 114: 1277-1285.

29. TABUNG FK, STECK SE, LIESE AD et al. Patterns of change over time and history of the inflammatory potential of diet and risk of breast cancer among postmenopausal women. Breast Cancer Research and Treatmente, 2017; 159(1): 139-149.

30. TORRES DX, LIMA ICS, CARNEIRO PCPDM. Associação entre gordura corporal e lipídios dietéticos de pacientes com câncer de mama. Revista Interdisciplinar, 2015; 8(1):1-8.

31. VAHID F, SHIVAPPA N, HATAMI M et al. Association between dietary inflammatory index (DII) and risk of breast cancer: A casecontrol study. Asian Pacific Journal of Cancer Prevention, 2018; 19(5): 1215-1221.

32. WIRTH MD, SHIVAPPA N, STECK SE et al. The dietary inflammatory index is associated with colorectal cancer in the National Institutes of Health-American Association of Retired Persons Diet and Health Study. British Journal of Nutrition, 2015; 113(11): 18191827.

33. WOOD I, TRAYHURN P. Adipokines and the signaling role of adipose tissue in inflammation and obesity. Future medicine, 2006; 1(1): 81-89.

34. WORLD HEALTH ORGANIZATION. The World Health Report - Global Strategy on Diet, Physical Activity, and Health. Geneva: WHO. 2004.

35. WU JT, WU LL. Linking inflammation and atherogenesis: Soluble markers identified for the detection of risk factors and for early risk assessment. Clin Chim Acta. 2006; 366:74-80.

36. ZHENG J, TABUNG FK, ZHANG J et al. Association between post-cancer diagnosis dietary inflammatory potential and mortality among invasive breast cancer survivors in the women's health initiative. Cancer Epidemiology, Biomarkers \& Prevention, 2018; 27(4): 454-463.

37. ZUCCHETTO A, SERRAINO D, SHIVAPPA N et al. Dietary inflammatory index before diagnosis and survival in an Italian cohort of women with breast cancer. British Journal of Nutrition, 2017; 117(10): 1456-1462. 